\title{
Die Vögel der Umgebung von Lissa i./P.
}

\section{Von C. Kayser.}

Die Umgegend von Lissa i./P., wo ich früher über 6 Jahre wohnte, ist ziemlich vogelreich, ja manche Örtlichkeiten, wie der Striesewitzer Wald und die Brüche, gehören zu den vogelreichsten Gegenden, welche ich überhaupt kennen gelernt habe.

Das Beobachtungsgebiet umfarst die Umgebung der Stadt Lissa i./P. bis zu einer Entfernung von etwa $10 \mathrm{~km}$. Es ist durchweg eben, nur im Nordosten der Stadt befindet sich etwas Hügelland, welches von dem Kankeler und Storchnester Walde bedeckt ist und dessen höchster Punkt, der „Hedwigsblick“ $150 \mathrm{~m}$ hoch ist.

Die Stadt Lissa selbst ist auf Lehmboden erbaut und infolge ihrer mit alten Bäumen bestandenen Promenade, ihrer Gärten und Friedhöfe eine der vogelreichsten Städte, welche ich kennen lernte. Die Promenade, welche die innere Stadt wie ein grüner Gürtel umgibt, hat eine solche Längenausdehnung, dafs man eine gute halbe Stunde braucht, um sie im gewöhnlichen Spaziergängerschritt zu durchschreiten. Ihre Breite beträgt durchschnittlich etwa 10-20 m. Ihre Anziehungskraft für die Vogelwelt wird dadurch erhöht, dafs sie grörstenteils von ausgedehnten Gärten mit einem reichen Bestande von alten Laubbäumen begrenzt wird. Dies gilt namentlich von den an die Ostpromenade anstofsenden, ehemals zu dem fürstlichen Schlofs gehörigen Gärten und von dem Schlofsplatz, dem ehemaligen Schlofspark, welcher eine Gröfse von etwas über 7 Morgen hat. Aufserdem weisen auch die drei in der Stadt belegenen christlichen Friedhöfe einen hübschen Bestand an alten Laubbäumen auf. Ferner haben sowohl die Promenade, als auch die oben erwähnten Gärten reiche Partieen jenes dichten Buschwerkes, welches für die Nachtigallen und Grasmücken unentbehrlich ist. So kommt es, dafs man auf dem Schlofsplatz, also mitten in der Stadt, den Gesang von Vögeln hört, welche man sonst nur in stillen Wäldern zu vernehmen gewöhnt ist, z. B. von Pirol, Zaunkönig, Schwarzplättchen, Waldlaubvogel u. s. w.

Von den Wäldern ist am nächsten gelegen, nur $1 \mathrm{~km}$ in südlicher bezw. südöstlicher Richtung entfernt, das ehemals fürstlich Sutkowski'sche, jetzt fiskalische Forstrevier Reisen, gegen 5000 Morgen grofs. Dasselbe ist fast ausschliefslich mit Kiefern, die auf magerem Sandboden stehen, bewaldet. Stellenweise sind Eichen, Birken, Fichten meist jüngeren Alters, Erlen u. s. w. eingesprengt. Unterholz findet sich nur an wenigen Stellen. Von den Charaktervögeln des Nadelholzes sind zwar die Haidelerche und der Triel, nicht aber die in andern Wäldern hiesiger Gegend anzutreffende Misteldrossel vorhanden. Wahrscheinlich sind der 
letzteren Vogelart die Bestände noch zu jung und es fehlen ihr auch zum grofsen Teil eingesprengte Wiesen. Überhaupt ist der Vogelbestaud in diesem Walde ein geringer, weil der Boden zu mager und die Kiefer ja überhaupt der den Vögeln am wenigsten zusagende Baum ist.

Dagegen stellt der Kankeler Wald, von der Stadt etwa $2 \mathrm{~km}$ in östlicher Richtung entfernt und gegen 4600 Morgen grofs, ein besonders schönes und auch vogelreiches Waldrevier dar, welches wegen seiner Mannigfaltigkeit und parkartigen Kultur einen Hauptanziehungspunkt für Naturfreunde bildet. Früher war es ein Eichenschälrevier und auch heute herrscht die Eiche noch im Revier vor. Nach ihr ist die Kiefer neben Lärche, Fichte, Buche, Kastanie, Aspe und ausländischen Nadelhölzern vertreten. Da es an alten Bäumen nicht mangelt und der Schwarzspecht und grofse Buntspecht für natürliche Nisthöhlen sorgen, sind auch die Höhlenbrüter vertreten.

Das Beobachtungsgebiet würde für den Oruithologen bei Weitem ergiebiger sein, wenn nicht in der $\mathrm{n}$ äh e r e n Umgebung der Stadt das Fehlen gröfserer Gewässer und die relative Trockenheit des Bodens entgegenständen. Abgesehen von unbedeutenden Wassergräben und einigen durch Lehmausstich entstandenen Tümpeln, sowie zwei kleineren Teichen, befindet sich noch in der Stadt auf dem Schlofsplatz ein künstlicher Teich, auf welchem weifse und schwarze Schwäne gehalten werden und halbwilde Stockenten sich aufhalten.

Im Anschlufs an den mit alten Bäumen bestandenen Gärten des Kaffeehauses Wolfsruhe befindet sich ein feuchter, aus jüngeren Erlen und anderen Laubbölzern gebildeter und gegen 21 Morgen grofser Busch und hinter demselben ein dicht bewachsener Teich. Diese Gegend würde noch weit vogelreicher sein, wenn sie nicht vom Publikum und namentlich von der Jugend nach allen Richtungen durchquert und belaufen würde. Hierzu kommen die häufigen Veränderungen des Geländes, da der Busch im Laufe der Jahre regelmälsig abgetrieben wird und sich durch Stockausschlag wieder bewaldet, auch manchmal ein Teil des Busches zu Feld eingeackert wird. Im Nachstehenden ist der Busch kurzweg als Erlenbusch bezeichnet.

Eine Folge der Wasserarmut ist die auffallende Tatsache, dafs die sonst so gemeine weifse Bachstelze (Motacilla alba) in der $\mathrm{n}$ äheren Umgebung der Stadt zu den seltenen Vögeln zählt.

Einen grofsen Vogelreichtum zeigen dagegen die in der hiesigen Gegend belegenen Brüche, allen voran der etwa $7 \mathrm{~km}$ von der Stadt in südwestlicher Richtung entfernte am polnischen Landgraben belegene und etwa 500 Morgen grofse Priebischer Bruch, der namentlich was das „kleine Zeug“, wie A. E. Brehm zu sagen pflegte, - anbetrifft, als ein Vogelparadies bezeichnet werden kann. Dieser Bruch ist vorwiegend mit Erle, aufserdem 
Aspe, Birke, Weide, Eiche, Esche und sehr vielem Buschwerk bestanden. Am Boden bildet Hopfen mit anderem Pflanzengewirr jenes undurchdringliche Dickicht, welches dem Vogelkundigen als Dorado mancher Rohrsänger- und Grasmückenarten bekannt ist. Dort singen der Heuschrecken- und der Flufsrohrsänger ihre eigenartigen Lieder nnd neben der Nachtigall kommt auch vereinzelt der Sprosser vor. Treten wir aber aus dem Walde auf die ihn umgebenden feuchten Wiesen, so hören wir die Pfiffe des grofsen Brachvogels (Numenius arquatus) und des Gambettwasserläufers Totanus calidris).

Dicht neben dem Priebischer Bruch erstreckt sich der etwa 2316 Morgen grofse Lauber Bruch, welcher mehr den gewöhnlichen Charakter unserer Laubwäldcr trägt und von grolsen Wiesen durchzogen ist. Hier ist das Brutrevier des Kranichs und Kornweihe.

Ferner ist $\mathrm{zu}$ nennen der früher zu dem Reisener Forstrevier gehörige, etwa 1200 Morgen grofse Tharlanger Bruch, welcher nach seinem Übergang in das Eigentum des Staates fast ganz abgeholzt und in Wiesen umgewandelt wurde. Nur ein kleiner, etwas über Hundert Morgen grofser Teil ist auf meine Anregung hin stehen geblieben und als Naturschutzpark in Aussicht genommen. Den Tharlanger Bruch durchschneidet der polnische Landgraben.

Dieses letztere Gebiet schliefst sich an den von Lissa i./P. etwa $6 \mathrm{~km}$ entfernten und 2400 Morgen grofsen Kraschener Bruch an, welcher hauptsächlich mit Erle und anderem Laubholz, aufserdem stellenweise mit Gruppen von Kiefern und Fichten bestanden ist. Am Kraschener Bruch geht der schlesische Landgraben her.

In Anschlufs an die Brüche will ich den Striesewitzer Wald erwähnen, der von der Stadt $3 \mathrm{~km}$ entfernt und in südwestlicher Richtung belegen ist. Seine Grölse beträgt etwa 2000 Morgen. $\mathrm{Er}$ ist teils mit Kiefern, teils mit Laubhölzern bestanden. Da die letzteren Partien viel Feuchtigkeit aufweisen und auch infolge der Tätigkeit der Spechte an Niststellen für Höhlenbrüter, wie Hohltaube und Blauraken, kein Mangel ist, stehen diese Waldesteile an Vogelreichtum den Brüchen nicht nach.

Auffallend ist in hiesiger Gegend das sehr spärliche Vorkommen der Fichte, welches seinen Grund teils in den Bodenverhältnissen, teils darin hat, dafs die Luft des für diesen Baum erforderlichen Grades von Feuchtigkeit ermangelt. Mit dem Mangel an Fichten hängt die geringe Zahl mancher Vögel, z. B. der Singdrossel, zusammen.

In weiterer Entfernung von der Stadt befinden sich mehrere gröfsere Gewässer, nämlich der Klein-Kreutscher und Storchnester See in einer Entfernung von je $9 \mathrm{~km}$ und der Retschker See in einer Entfernung von $12 \mathrm{~km}$.

Was die Beschreibung der Vogelimmen anbetrifft, so gab ich der Wiedergabe in Silben den Vorzug. Sie bat 
zwar entschieden ihre Mängel, aber derjenige, welcher die betreffenden Vogelstimmen einmal gehört hat, kann sie sich und Anderen durch richtiges Aussprechen der Silben recht wohl versinnlichen. Die Wiedergabe in Noten ist nur bei wenigen, hierzu besonders geeigneten Vogelstimmen (wie Pirol, Amsel u. a.) möglich. Die Wiedergabe anderer Vogelstimmen in Noten mag zwar ein recht beachtenswertes musikalisches Kunststück sein, ist aber nicht recht bezeichnend, denn musikalische Noten oder die von Voigt eingeführten Schriftzeichen geben lediglich die Tonhöhe und den Rhytmus wieder, während für Vogelstimmen gerade die Klangfarbe besonders charakteristich ist. Übereinstimmend hiermit urteilte über diese Fragen auch der verstorbene Stolz (vrgl. „Über die Vogelwelt der Oberlausitz etc." in den "Abhandlungen der Naturforsch. Gesellschaft zu Goerlitz" 1911 S. 4).

$\mathrm{Zu}$ Beobachtungen benutzte ich einen guten Galilei'schen Feldstecher, in den letzten Jahren auch den Prismen-Feldstecher Binolas $6 \times$ von Leitz in Wetzlar, welcher mir sehr gute Dienste leistete.

Eine sorgfältige Tagebuchführung war selbstverständlich für meine Beobachtungen unerlä sslich. Ich babe indessen darauf verzichtet, vollständige Tagebuchauszüge $\mathrm{zu}$ veröffentlichen, wie dies nicht selten geschieht, - weil hierdurch meines Erachtens die Beobachtungsliteratur in unnötiger und unzweckmäfsiger Weise anschwillt. In der systematischen Anordnung und in der Nomenklatur bin ich im wesentlichen dem „Systematischen Verzeichnis der Vögel Deutschlands" und den „Kennzeichen der Vögel Deutschlands" von Geheimrat Prof. Dr. Reichenow gefolgt. Auf das Verzeichnis bin ich namentlich da zurückgegangen, wo es sich um die Vermeidung eines Doppelnamens handelte.

Für die Mafse und die dafür gebrauchten Abkürzungen war das Reichenow'sche Verfahren (Journ. f. Ornith. 1891 S. 346 ff.) malsgebend.

Da ich bereits in der Zeitschrift der naturwissenschaftlichen Abteilung der Deutschen Gesellschaft für Kunst und Wissenschaft in Posen (Heft 70,71, 73,78 u. 80) ornithologische Beobachtungen aus hiesiger Gegend veröffentlicht habe, liefsen sich Wiederholungen nicht vermeiden. Ich hatte auch dagegen um so weniger Bedenken, als die genannte Posener Zeitschrift in den Kreisen der Ornithologen nur wenig verbreitet ist.

Aufgeführt sind diejenigen Arten, welche in einem Umkreise von etwa $15 \mathrm{~km}$ von der Stadt gerechnet, betroffen wurden.

1. Erithacus philomela Bchst. Den $\mathrm{Sp}$ ross er habe ich nur in einem Stück beobachtet. Ein ơ schlug am 26. V. 1914 im Priebischer Bruch an einer besonders nassen Stelle auf einer Erle. Der Vogel hatte nicht jenen ,verfitzten"Schlag ohne Caesur, wie ihn manche Sprosser zum Vortrag bringen, sondern einen schön en 
charakteristischen Schlag mit einem der Singdrossel ähnlichen Rhytmus und sprechenden Rufen. Nach Mitteilung des H. Lehrers Hausdorf in Priebisch hat auch im Jahre 1916 ein Sprosser im dortigen Bruch geschlagen und zwar nach der Beschreibung zu schliefsen - an derselben Stelle wie damals. Sonst kam im Priebischer Bruch nur die Nachtigall zur Beobachtung.

2. Erithacus luscinia L. Die $\mathrm{Nachtigall}$ ist in der hiesigen Gegend ziemlich häufig und auch in der Stadt eher im Zunehmen als im Abnehmen begriffen. Im städtischen Weichbild zählte ich 1913 mindestens 8, 1916: 11 und 1917: 15 schlagende $\sigma^{\top} \sigma^{\top}$. - In den 6 Jahren 1913-18 war ihr frühestes Ankunftsdatum der 21. IV., das späteste der 2. V. und das durchschnittliche der 27. IV. - Am 15. V. 16. Gelege von 5 Eiern. Da dasselbe, wohl infolge von Nässe, verlassen war, nahm ich es am 30. V. 16. fort.

Mafse von 4 dieser Eier: $22 \times 15,5,21,5 \times 15,5,21 \times 15$, und $21 \times 15$. Die Eier waren schwach bebrütet, übrigens ungleich entwickelt.

Den letzten Schlag hörte ich 1916 am 4. VII, 1917 am 9. VII. und 1918 am 18. VII. - 1918 sah ich die letzte Nachtigall am 10. VIII. Die Nachtigall hält sich während der Brutzeit auch regelmäfsig auf den hiesigen Friedhöfen auf, obwohl dieselben wenig Strauchwerk, vielmehr neben alten Laubbäumen und Lebensbäumen nur dichte Bodengewächse, namentlich Epheu, aufweisen. Die Häufigkeit dieser Vogelart in hiesiger Gegend beweist, dafs sie sich auch mit wasserarmem Gelände abzufinden versteht.

3. Erithacus rubeculus L. Das Rotkehlchen ist hier ziemlich bäufig, es kommt insbesondere im Reisener und Kankeler Walde, im Priebischer Bruch und auch auf der Promenade vor. Ein Rotkehlchen überwintert hier regelmäfsig im Gymnasialgarten und ist dann auch das erste, welches Ende März oder Anfang April seinen Gesang ertönen läfst. 1914 beobachtete ich das erste am 1. IV., 1918 am 9. IV. Der Durchzug fand 1918 vom 6. X. bis Ende Oktober statt.

4. Erithacus cyaneculus Wolf. Das Blaukehlchen wurde nur einmal, nämlich von $\mathrm{H}$. Förster Paul-Lafswitz, dem der Striesewitzer Wald untersteht, vor 5 bis 6 Jahren unweit seines Wohnhauses beobachtet. Sonst kommt dieser Vogel hier nicht vor.

5. Erithacus phoenicurus L. Der G arte $\mathrm{nr}$ ot s c h w a n z ist hier häufiger Brutvogel in den Wäldern, Friedhöfen, Promenaden und gröfseren Gärten. In den 4 Jahren 1913 und 1915-1917 war das früheste Ankunftsdatum der 12. IV., das späteste der 20. IV. und das Durchschnittsdatum der 17. IV. Den letzten Gesang hörte ich 1914 am 13. VIII. Ein $\sigma^{\top}$ hatte Ähnlichkeit im Gesang mit dem in seiner Nachbarschaft ansässigen Trauerfliegenschnäpper. 
Am 17. V. 16. Gelege von 4 oder 5 Eiern. Flügge Junge am 13. VI. 13., 17. VI. 16., 24. VII. 17. und 25. VI. 18. Am 17. VI. 18. zeigte mir H. Lehrer Schittel hier das Nest eines Gartenrotschwanzes am Fenster des zweiten (bezw. dritten) Stockes der evang. Volksschule in einem Futterhäuschen erbaut. Das letztere hatte vorn Einflugslöcher; die hintere Glaswand, welche an der Fensterscheibe stand, war bis auf eine kleine Öffnung mit Papier verklebt. Trotzdem es sich am Fenster eines Klassenzimmers befand, fütterten die alten Vögel ungestört. H. Schittel konnte sogar den Deckel des Häuschens hochheben und wieder aufsetzen. Die Jungen waren halbflügge.

6. Erithacus titys L. Der $\mathrm{Hausrots} \mathrm{chwanz}$ ist etwas weniger zahlreich als der Gartenrotschwanz, - in manchen Jahren, wie 1916, bäufiger als früher. An Kirchen und hohen Gebäuden für seinen Aufenthalt fehlt es hier nicht, aber vielleicht stehen die zahlreichen Dohlen seiner Zunahme im Wege. In der hiesigen Ansiedlung hat er sich auch niedergelassen, obwohl es dort nur niedrige, einstöckige, allerdings massive Häuser gibt. 1915 Ankunft am 5. IV., 1916 am 2. IV., 1918 am 8. IV. Am 18. VI. 15. in Lafswitz flügge Junge. 1917 hörte ich den Hausrotschwanz schon vom 14. IX. ab wieder singen. Einen abweichenden Gesang hörte ich in der Morgendämmerung am 15. VI. 16. Der Sänger brachte die krächzende Gurgelstropfe nicht heraus, sang statt deren eine andere Stropfe. Die nächste, pfeifende Stropfe sang er dann wieder normal.

7. Pratincola rubetra L. Das $\mathrm{B} \mathrm{ra} \mathrm{un} \mathrm{ke} \mathrm{hl} \mathrm{ch} \mathrm{en} \mathrm{ist} \mathrm{in}$ hiesiger Gegend nicht selten. Ein of sang genau wie Sylvia rufa, so dafs ich glaubte, der Gesang rühre von diesem Vogel her und mich erst der Augenschein über meinen Irrtum aufklärte.

8. Saxicola oenanthe $\mathrm{L}$. Der $\mathrm{St}$ e in s c h m ät $\mathrm{z}$ e r kommt zuweilen vor. Am 23. VI. 1915 beobachtete ich Junge von demselben Jahr.

9. Turdus merula L. Die A $\mathrm{m} \mathrm{sel}$ ist hier häufiger Brutvogel. . In der Stadt singt mindestens ein halbes Dutzend Männchen im Frühjahr in den Promenaden, Friedhöfen und grölseren Gärten. Nach einer mir gemachten zuverlässigen Mitteilung ist die Amsel etwa seit 1905 oder 1906 in die Stadt Lissa i./P. dauernd eingerückt.

Anders liegen die Verhältnisse in der Stadt Posen. Prof. Hammling sagt darüber (Jourii. f. Ornith. 1911 S. 580) „Während etwa bis 1907 die Amsel nur recht vereinzelt in der Umgebung Posens zu finden war, hat sie sich seit dieser Zeit erheblich vermehrt und war nunmehr an allen geeigneten Plätzen in einem oder auch mehreren Pärchen vertreten, ohne dafs sie sich jedoch bisher zu einer eigentlichen Stadtamsel entwickelt hätte." Dagegen schreibt Hammling später (Journ. f. Ornith. 1918 S. 210: 
„Es scheint eine Umbildung unserer Park- zur Stadtamsel im Wege zu sein." - In den Wäldern ist die Amsel hier entschieden zahlreicher als die Singdrossel, währerd in den schlesischen Forsten, welche ich kenne, das umgekehrte Verhältnis herrscht. 1913 erster Gesang am 13. III., 1914 am 27. II. Auffallender Weise sang ein $\sigma^{\top}$ am 2. und 3. XI. 15. auf der Promenade laut wie im Frübjahr und machte eine Tour des Singdrosselschlages täuschend nach, die es wohl von der Singdrossel eines Nachbargartens - der einzigen, welche sich hier in der Stadt ansiedelte und welche in späteren Jahren sich nicht mehr zeigte, - erlernt hatte. Die hiesigen Amseln sind gute Sänger, von einer Entartung des Gesanges, wie ich sie an einer Stadtamsel in Wien bemerkte, ist bei ihnen keine Spur wahrzunehmen.

Ein Nest stand in dem Stachelbeerstrauch eines Gartens kaum $1 \mathrm{~m}$ hoch, ein anderes im Erlenbusch zwischen mehreren aus gemeinsamer Wurzel entspringenden jungen Erlenstämmen etwa $30 \mathrm{~cm}$ über der Erde. Auf dem hiesigen evang. Friedhof baut die Amsel ihre Nester regelmäfsig auf Lebensbäumen in doppelter bis dreifacher Mannshöhe. 1915 ist nach glaubhafter Mitteilung des Friedhofsgärtners Busko eine Brut junger Amseln schon in der letzten Aprilwoche ausgeflogen. - 1917 hörte ich eine noch am 18. VII. fleifsig singen. - Am 14. I. 17. frafsen drei Amseln die Beeren des wilden Weins an einem Hause der Kaiser Wilhelmstrafse. Im Winter ist die Zahl der hier verweilenden Amseln gröfser als im Sommer, sie müssen entweder durch die Amseln der hiesigen Wälder oder durch Artgenossen aus anderen Gegenden verstärkt worden.

10. Turdus musicus L. Die Singdrossel kommt in beschränkter Zahl in den benachbarten Wäldern vor. 1915 war sie-spärlicher vertreten als vorher und seitdem hat sie noch weiter abgenommen. Sie bewohnt auch ältere Kiefernschonungen. Als Ursachen der Abnahme können die Wiederfreigabe des Dohnenstriches und die Überzahl der Eichbörnchen in Betracht kommen. Allerdings fehlt dieser letztere Grund in Trachenberg in Schlesien und auch dort bemerkte ich ihre Abnahme. Ein am 11. X. 12 im Walde gefundener Federnkranz schien dieser Art anzugehören. Am 7. X. 12. wurde ein am Telegraphendraht verunglücktes Stück eingeliefert. Herr Forstmeister Dommes schofs einen Sperber $\sigma^{\top}$, welcher die Reste einer Singdrossel verzehren wollte. Nur einmal bewohnte eine Singdrossel - im Jahre 1915 - einen Garten der Stadt. In späteren Jahren ist sie dorthin nicht zurückgekehrt. - 1914 Ankunft am 24. III., 1915 am 30. III.

11. Turdus iliacus L. Die Rotdrossel ist hier als Durchzugsvogel nicht selten. Am 13. und 14. X. 1914 zogen sie hier durch. Sie waren, wie ich dies auch früher in Oberschlesien bei dieser Art beobachtete, sehr unruhig und scheu, salsen stets 
auf den höchsten Bäumen und lockten ssrii, genau wie die Amsel. Am 11. IV. 16. liefs eine Rotdrossel im Priebischer Bruch zuerst ihren leisen Gesang und dann die laute, charakteristische Decrescendo-Flötenstropfe hören.

12. Turdus visicivorus L. Mehrere singende $\sigma^{x} \sigma^{x}$ der M is teld rossel beobachtete ich zur Brutzeit im Striesewitzer Walde. In der weiteren Umgebung kommt sie auch in dem etwa $16 \mathrm{~km}$ entfernten Luschwitzer Forst ziemlich häufig vor. Ein an einem Telegraphendraht verunglücktes Stück wurde am 7. X.12. eingeliefert und ferner ein ebensolches im Frübjahr 1918.

13. Turdus pilaris L. Einen Flug der $\mathrm{W} \mathrm{a} \mathrm{cholder-}$ drossel beobachtete ich kurz vor Weihnachten 1915 auf der Ostpromenade. Der Ausstopfer H. Lenhard traf sie auch in den mit Laubholz bestandenen Rändern des Kankelers Waldes.

14. Regulus cristatus Vieill. Das g e l b k ö p fig e Gold. $\mathrm{h} a ̈ \mathrm{~h} \mathrm{n} \mathrm{ch} \in \mathrm{n}$ ist ziemlich häufig.

15. Phylloscopus rufus Bchst. Der Weiden laubsänger ist häufig. Bär und Stolz nennen den Fitislaubsänger für die proufsische Oberlausitz den verbreitetsten seines Geschlechtes. In der hiesigen Gegend dürften beide Arten etwa an Zahl einander gleichstehen. Man trifft den Weidenlaubsänger im gemischten Hochwald, auch ist er ständig auf den hiesigen Friedhöfen und in grölseren Gärten vertreten, wo er auch brütet. In den Jahren 1913-1917 war das früheste Ankunftsdatum der 29. III., das späteste der 12. IV., das durchschnittliche der 4. IV. - Auf den Friedhöfen nistet er meistens im Epheu der Gräber. Beim zu Nest tragen rifs ein Weidenlaubsänger Niststoff aus einem alten Amselnest. Am 6. V. 1917 trug einer noch um 111/. Uhr Vorm. zu Nest. Ein Nest, welches $10 \mathrm{~cm}$ über dem Erdboden in einem Stachelbeerbusch stand, enthielt am 5 VI. 17: 4 Eier. Ein anderes Nest stand in einem Garten der Stadt ebenfalls in einem Stachelbeerstrauch und enthielt am 25. VII. 17. kleine Junge. Es war sehr lang und röhrenförmig, die Jungen ganz im Hintergrund. H. Steuerinspektor Romeils fand im Kreise Schmiegel ein Nest des Weidenlaubsängers in einem Brombeergebüsch $62 \mathrm{~cm}$ ü b e r d e r E rd e. Es enthielt am 25. VII. 17. etwa 5 Eier und am 5. VIII. 17. die frisch ausgeschlüpften Jungen. Dieses Nest, welches ich zu untersuchen Gelegenheit hatte, war ebenfalls ziemlich lang und röhrenförmig, vom Eingang bis zur Hinterwand innen etwa $10 \mathrm{~cm}$ lang. Interessant war mir, dafs zum Nestbau sehr viel Kiefernrindenplättchen verarbeitet waren, was ich bei diesem Vogel noch nie sah. Ein anderes Nest fand derselbe Beobachter in einem Wachholderstrauch ge na u 1 m über dem Erdboden.

Der Gesang ertönt bis in den Juli (z. B. am 4. VII. 15. und 27. VII. 16.) und beginnt dann wieder im September (z. B. 
9. IX. bis 25. IX. 17.) 1918 hörte ich noch sogar am 16. X. einen singen, dessen Gesang recht unbehilflich klang, es war wohl ein junger Vogel.

Ich hörte einige merkwürdig abweichende Sänger: Am 29. V. 16. hörte ich einen Weidenlaubsänger, welcher zwischen den gewöhnlichen einsilbigen Lauten (zilp zalp) zweisilbige einreihte, etwa wie zilp zilp ziddid zilp zilp ziddid zilp zilp. Wohl denselben Vogel hörte ich auch am 25. und 27. VII. 16. Sein Gesang klang da wie zizizilp zizizilp (also dreisilbig), dann aber zilp zilp - wie gewöhnlich - weiter. Ich mufs bemerken, dafs ich ebenso wie v. Loewis (N. Nauman II. S. 105 Anm. 1) in dem Gesang dieses Vogels ein deutliches $\mathrm{z}$ oder ts höre. Dio Naumann'sche Wiedergabe mit dilm delm u.s. w. finde ich nicht bezeichnend. Spreche ich mir dagegen die Silben zilp zalp u.s. w. in dem richtigen Rhytmus vor, so höre ich im Geiste den kleinen Hämmerer. Einen im Gesang ähnlich abweichenden Vogel, wie der oben geschilderte, hörte ich im Jahre 1917 auf der Ostpromenade. Ein Weidenlaubsänger sang am 28. IV. 17. auch noch bei einer Temperatur von $+4^{\circ} \mathrm{R}$.

Am 15. VIII. 15. lag ein Weidenlaubsänger, anscheinend durch Anfliegen an einem Draht verunglückt, tot auf der Strafse. Das Gelb an der Unterseite und an den inneren Flügeldeckfedern erschien schwefel- (nicht ocker-) farbig. Der Vogel war in der Mauser und hatte an Rücken, Hals und innerem Flügelbug frische, noch kielige Federn. Long. tota: $11,2 \mathrm{~cm}$. Ala infra mens. $65 \mathrm{~mm}$. Rostr. a. fr.: $9 \mathrm{~mm}$. Der Ausstopfer erhielt Anfangs September 1915 gleichfalls einen am Draht verunglückten Weidenlaubsänger.

Al sich am 22. IV. 16. auf Birkhähne ansals, kam einer dieser zutraulichen Laubsänger in meinen aus Weidensträuchern und jungen Kiefern gebildeten Schirm und mir so nahe, dafs ich ihn mit meinem Spazierstock hätte treffen können. 1918 traf ich noch am 23. X. auf der Promenade einen Weidenlaubsänger an, der nur lockte, nicht sang.

Malse von 4 Eiern: $15,5 \times 11,5-15 \times 11,5-15,5 \times 11$ und $15,5 \times 11$. Sie stammen aus e i $\mathrm{n} \mathrm{e} \mathrm{m} \mathrm{Gelege.}$

16. Phylloscopus trochilus L. Auch der Fitisla u b sänger ist hier häufig. In Feldhölzern mit jüugeren Birken und im Kiefernstangeuholz. Im April nach der Ankunft singt er manchmal in den Gärten und Friedhöfen der Stadt, zieht sich aber dann zur Brutzeit in die Wälder. Nur einen einzigen hörte ich zur Brutzeit (z. B. am 9. V. 15.) auf dem Schlofsplatz und in einem diesem benachbarten Garten singen, wo er auch vielleicht genistet hat. - In den Jahren 1913-1917 war der erste Ankunftstag der 6. IV., der letzte der 1. V. und der Durchschnittliche der 17. IV. 
Den Baumlaubvogel (Phylloscopus sylvestris Meisner) habe ich, so sehr ich auch darauf achtete, nicht beobachten können. Sehr interessant ist die Arbeit von Dr. Gengler über ihn (Orn. Monatsschr. 1905 S. 556 ff.). Den Beweis, dafs diese Vögel lediglich imitierende Fitislaubsänger sind, halte ich keineswegs für geführt, eher würde ich an die Möglichkeit einer Bastardierung zweier so sehr nachstehender Arten wie $P h$. rufus und trochilus denken.

17. Phylloscopus sibilator Bchst. Der Waldla ub sänger ist hier weniger häufig als die beiden vorhergehenden Arten, aber immerhin ziemlich zahlreich, obwohl seine Lieblingsbäume, Fichte und Buche, in hiesiger Gegend schwach vertreten sind. Immerhin sagen ihm die jüngeren Mischbestände des Kankeler Waldes sehr zu und in dem nahen Schiefswerderwalde, welcher infolge seines mageren sandigen Bodens nur einen geringen Vogelbestand aufweist, bewohnt er alljährlich eine Stelle, wo Kiefer, Birke und Aspe in verschiedenen Altersstufen gemischt stehen. Nach der Ankunft im April hält er sich auch in der Stadt in den alten Schlofsgärten auf. - In den Jahren 1914 und 1916 notierte ich seine Ankunft am 23. IV., 1915 am 22. IV.

18. Hypolais philomela Rchw. Der Garten sänger ist hier häufig. In den Jahren 1913-1917 war das früheste Ankunftsdatum der 30. IV., das späteste der 9. V. und das Durchschnittliche der 5. V. - 1913 hörte ich den ersten Gesang am 7. V. trotz kalten Wetters (etwa $8^{\circ}$ R.). Einer trug das wäd wäd u. s. w. der Dorngrasmücke täuschend vor. Ein anderer brachte Kohlmeisenmotive, eigenartig zu Strophen verarbeitet. Ein Gartensänger, welcher im Erlenbusch in der Nachbarschaft des Sumpfrohrsängers (Acrocephalus palustris Bchst.) seinen Stand hatte, zeigte im Gesang grofse Ähnlichkeit mit dieser Vogelart. Der Gesang des Gartensängers ertönt bis in den Juli hinein (z. B. 6. VII. 15, 9. VII. 16, 11. VII. 18). - Am 10. VI. 13 ein Nest mit 5 Eiern in einem Hollunderstrauch (Sambucus niger) etwa $1 \mathrm{~m}$ hoch. Am 29. V. 14. ein Nest in einem Strauch und an eine Erle angelehnt, sowie durch Hopfen u. s. w. gut gedeckt, etwa $1 \mathrm{~m}$ hoch mit 1 Ei. Am 3. VI. 15. drei Nester mit Eiern belegt, von denen 2 in Mannshöhe standen, das dritte etwa $1 \mathrm{~m}$ über dem Boden. Am 18. VI. 16. Gelege von 5 Eiern. Auffallend war, dafs die Eier auch nach dem Beginn der Bebrütung öfters unbesetzt waren. Trotzdem kamen die Jungen glücklich aus. Ein zweites Nest in Augenhöhe enthielt am 3. VII. 16. kleine Junge, die leider bald darauf ausgeraubt wurden. Es stand etwa $1 \mathrm{~m}$ hoch in einem Sambucusstrauch.

19. Locustella naevia Bodd. Der $\mathrm{H}$ e u s c h r e cke $\mathrm{n}$ f ä n ge r ist an geeigneten Örtlichkeiten ziemlich häufig. Am 26. V. 14. hörte ich zwischen 6 und 10 Uhr Vorm. eine Anzähl Männchen im Priebischer Bruch. Am 14. V. 15. schwirrten im Tharlanger 
Bruch $30^{x} 0^{x}$, am 1. VI. 15. im Priebischer Bruch ebenfalls etwa 3. Im Jahre 1917 hörte ich ihn im Priebischer Bruch gar nicht, wohl weil der Bruch noch lange in Frühjahr unter Wasser stand.

20. Locustella fluviatilis Wolf. Der F l u fsroh rsänger ist in manchen Jahren häufiger als die vorhergehende Art, in anderen Jahren ist das Verhältnis umgekehrt. Am 26. V. 14. Vorm. zwischen 6 und 10 Uhr hörte ich auch von dieser Art eine Anzahl Sänger im Priebischer Bruch. Am 1. VI. 15. hörte ich etwa $3 \sigma^{\top} \sigma^{\top}$ daselbst. Ein $\sigma^{\top}$ sang sogar im Erlenbusch, dicht an der Stadt. 1917 hörte ich im Priebischer Bruch nur ein $\sigma^{x}$, wohl infolge des $\mathrm{zu}$ hohen Wasserstandes.

21. Acrocephalus palustris Bchst. Der $\mathrm{Sumpfrohr}$ $\mathrm{s}$ ä $\mathrm{n}$ g e $\mathrm{r}$ ist in mäfsiger Zahl vertreten im Erlenbusch an den dortigen Wassergräben, im Priebischer Bruch u. s. w. Er ist der am spätesten zurückkehrende Zugvogel. 1915 beobachtete ich am 1. VI. den ersten im Priebischer Bruch, im Erlenbusch gar erst am 3. VI., wohl weil die Vegetation infolge der Kälte und der grofsen Trockenheit sehr spät entwickelt war. Im Erlenbusch hatte ich vorher häufig nach ihm gesucht. 1916 hörte ich den ersten am 14. V., 1917 beobachtete ich den ersten am 22. V. Einer gab den Lockton der Kohlmeise und des Gelbspötters deutlich wieder.

22. Acrocephalus streperus Vieill. Der Teichrohrs änger kommt hier nur vereinzelt vor, ist aber Brutvogel. Sowohl die typische Form wurde von mir beobachtet als auch die Form horticolus. 1916 sangen $2 \sigma^{\top} \sigma^{\top}$ in bezw. am Erlenbusch. Einer davon hatte seinen Stand in einem Kornfeld und trug einen bei Weitem weicheren und wechselvolleren Gesang vor, als diese Vögel sonst haben. Er gehörte zweifellos der Unterart horticolus an. Auch Kleinschmidt hörte diese in Kurhessen im Korn singen (N. Naumann II. S. 76). Sein Nest hatte dieser Gartenrohrsänger ebenfalls im Korn und zwar war es in derselben Weise zwischen die Stengel einer Distel gewoben, wie der typische streperus dies im Rohr zu tun pflegt und âus trockenen Halmen, Würzelchen und einigen Blättern mit ziemlich flachem Napf erbaut. Im Frühjahr 1917 war sein früherer Standort für ihn verloren, weil an Stelle des damaligen Roggenfeldes nur niedrige, grüne Feldfrucht vorhanden war, in der er sich nicht ansiedeln konnte. Er hielt sich daher in dem nahen Laubholz des Erlenbusches auf. Im Gesang hatte er unter Anderem auch eine dem ihm benachbarten Gartensänger sehr ähnliche Tour, während doch sonst die Gesänge des echten streperus uud der Hypolais philomela grundverschieden sind. Ein streperus sang am 18. VI. 17 noch unermüdlich.

23. Acrocephalus arundinaceus L. Der D rosselrohrs ä $\mathrm{g} g$ er ist spärlich vertreten, so am Storchnester See, an einem 
kleinen Teich im Lauber Bruch und neuerdings seit 1917 auch bei der hiesigen Badeanstalt an dem Wassergraben, wo sich kleine Rohrhorste gebildet haben. An letzterem Ort safs er während des Gesanges ungedeckt in den obersten Zweigen einer Weide und liefs sich ohne Scheu beobachten. 1918 hörte ich ihn zuerst am 1. V.

24. Sylvia atricapilla L. Die Mö $\mathrm{n} \mathrm{h} \mathrm{s} g \mathrm{ra} \mathrm{sm} u ̈ \mathrm{cke}$ ist hier die häufigste Grasmücke, auch auf der hiesigen Promenade und den Friedhöfen u. s. w. zahlreich vertreten. In den Jahren 1913-17 war das erste Ankunftsdatum der 25. IV., das letzte der 4. V., das durchschnittliche der 28. IV. Am 25. V. 14. in einem Gesträuch etwa $0,5 \mathrm{~m}$ hoch ein Nest mit einem Ei. Am 29. V. 14. brütet der Vogel darauf. Au 11. VI. 15. Gelege von 5 Eiern. Der Gesang ertönt bis Mitte Juli (16. VII. 15, 15. VII. 16, 9. VII. 17.) Auch am 26. VIII. 14. und 5. IX. 18. hörte ich auf der Promenade ihren halblauten und bezw. mehr als halblauten Schlag. Die hiesigen Mönchsgrasmücken sind recht gute Sänger, welche kraftvolle Schläge von erheblicher Jänge vortragen. Ein am Draht verunglücktes ơ mals A. i. m. $78 \mathrm{~mm}$.

25. Sylvia curruca L. Die $\mathrm{Z}$ a u $\mathrm{g} \mathrm{r}$ a s mü cke ist hier wohl die am wenigsten häufige Grasmücke. Auf Promenaden, in Dorfgärten, im Walde auch in Kiefernschonungen. - In den Jahren 1914-1917 erster Ankunftstag der 12. IV., letzter der 1. V., durchschnittlicher der 21. IV. An Zahl scheint sie in den letzten Jahren etwas zugenommen zu haben. Am 9. V. 15. baute eine Zaungrasmücke auf dem kath. Friedhofe an ihrem Nest, $\% \mathrm{~m}$ hoch in einem Buchsbaumstrauch. Am 13. V. erstes Ei. Leider verschwand das Nest auf unaufgeklärte Weise.

26. Sylvia rufa Rchw. Die D o rngrasmü cke findet sich im Erlenbusch, wo sie auch brütet. und an geeigneten Stellen in den Wäldern (Waldränder und noch junge, nicht dichte Kieferschonungen). Seit dem Frühjahr 1917 kommt sie auch auf der biesigen Promenade vor. Ein $\sigma^{*}$ safs wiederholt in der Krone eines ziemlich hohen Baumes der Promenade oder auf einem benachbarten Telegraphendraht und sang daselbst. Diese Art hat in den letzten Jahren zugenommen. In einem Nest am 3. VI. 15. kleine Junge. Am 19. VI. 16. Gelege von 5 Eiern. Dieses Gelege war auffallender Weise während der Bebrütung manchmal unbesetzt.

27. Sylvia simplex Lath. Die Gartengras mü $\mathrm{cke}$ ist hier an geeigneten Orten viel weniger zahlreich, als ich sie in früherer Zeit in Niederschlesien zu finden pflegte. Im Erlenbusch fehlt sie seit einiger Zeit. In mälsiger Zahl bewohnt sie den Priebischer Bruch. Im Frübjahr 1918 hatten sich auch $2 \sigma^{\top} \sigma^{\top}$ auf der hiesigen Ostpromenade eingestellt, wo sie früher fehlte. Sie nistet zwar in Sträuchern, hält sich aber mit Vorliebe auf Laubholzbäumen in mittlerer Höhe auf und singt auch dort. 
28. Sylvia nisoria Bchst. Die S p e r b er g r a s m ü ck e kommt im Priebischer Bruch, an Waldrändern und auch in Feldsträuchern vor, welche in der Nähe von Baumalleen stehen. Im Gegensatz zur vorigen Art liebt sie höhere Sträucher, besonders Dornsträucher, mehr als Bäume. Stolz (Abh. der naturforsch. Gesellschaft zu Goerlitz 1911 S. 63) spricht von der Sperbergrasmücke als einem „Freunde f e u chten (von mir unterstrichen) und dichten Buschwerks" und Rich. Schlegel spricht sich sogar (J. f. Orn. 1918 S. 46) foldendermafsen aus: "Aber auch in den geeignetsten Hecken wird sich diese stattliche Grasmücke nicht dauernd niederlassen, wenn sich nicht wenigstens ein Bewässerungs- oder Abzugsgraben dort, ein Flufslauf resp. eine Lache daselbst befinden".

Demgegenüber mufs ich betonen, dafs ich die Sperbergrasmücke sowohl früher in Schlesien, als auch jetzt in hiesiger Gegend öfters an völlig trockenen Orten traf, nur das dichte nicht zu niedrige Buschwerk fehlte ihrem Standort niemals. Hier in Lissa i./P. beobachtete ich sie auch in den Gärten des Landrahtsamtes und auf dem evang. Friedhof. Mitte Mai 1913 verunglückte ein Stück an einem Drath. Ihr Gesang ähnelt so aufserordentlich dem von $S$. simplex, dafs selbst mein geübtes $\mathrm{Ohr}$ sich schon manchmal getäuscht hat. Kürzere Strophen und häufiger wechselnde Tonhöhe sind für die Sperbergrasmücke charakteristisch, am sichersten ist sie an ihrem Schnarren kenntlich.

29. Accentor modularis L. Die $\mathrm{Hecke} \mathrm{nbraunelle}$ habe ich nur einmal - offenbar auf dem Durchzuge - am 12. IV. 17. in Wolfskirch beobachtet.

30. Troglodytes parvulus Naum. Der $\mathrm{Z} \mathrm{a} \mathrm{u} \mathrm{n} \mathrm{k} \mathrm{ö} \mathrm{n} \mathrm{ig} \mathrm{bat} \mathrm{in}$ den letzten 6 Jahren erheblich abgenommen, ohne dafs ich den Grund angeben kann, falls nicht etwa die Katzen daran schuld sind. Im Bezirk der Stadt ist er nur noch selten anzutreffen, dagegen hält er sich in den Brüchen auf.

31. Aegithalus caudatus L. Die $\mathrm{S} \mathrm{chwanzmeise} \mathrm{ist}$ hier etwas spärlich vertreten. Ich habe nur die weilsköpfige Form beobachtet. In Laubholzpartien, selten (wie am 25. VIII. 18.) auch im reinen Kiefernbestand. Ich traf diese Art in $\mathbf{F a -}$ milien, auch Stücke im Jugendkleide, vermag aber nicht mit Sicherheit zu behaupten, dafs sie hier Brutvogel ist. Ihr Lockton sisisi $(2-5 \mathrm{mal})$ hat grofse Âhnlichkeit mit dem Lockton des gelbköpfigen Goldhähnchens.

32. Parus cristatus mitratus Brehm. Auch die $\mathrm{H}$ a u be n$\mathrm{mei}$ s e ist ziemlich spärlich und nur im Kiefernwalde vertreten.

33. Parus palustris subpalustris Brehm. Die $\mathrm{Su} \mathrm{m} \mathbf{p f -}$ $m \theta$ i s e ist hier nächst der Kohlmeise wohl die häufigste Meisenart. Sie ist auch im Stadtbezirk bäufig und besucht im Winter 
die Futterkästen auf den Fenstern. 1917 erster Gesang am 14. III.

Die Form salicarius konnte ich hier nicht auffinden.

34. Parus ater $\mathrm{L}$. Die $\mathrm{T}$ a $\mathrm{n} \mathrm{n}$ e $\mathrm{n} \mathrm{m} \theta$ is e kommt hier nur sehr spärlich vor. So beobachtete ich sie am 29. IX. 18, wie sie in Gemeinschaft mit der Haubenmeise Kiefernsamen auflas, der aus den von Eichbörnchen zerschroteten Zapfen gefallen war. Ihre Lockrufe ähneln der Kohlmeise, klingen aber viel weicher.

35. Parus caeruleus L. Die B l a u $\mathrm{m}$ e i s e ist hier häufig. Sie brütet auch mitten in der Stadt auf der Promenade und im Walde manchmal in künstlichen Nisthöhlen. Am 25. V. 13. ffügge Junge. 1917 brütete im Priebischer Bruch ein Paar im Loch einer Erle. etwa $70 \mathrm{~cm}$ hoch vom Boden und hatte am 9. VI. Junge.

36. Parus major L. Die $\mathrm{Kohlm}$ e ise ist wie überall, so auch hier die häufigste Meisenart. 1915 erster Frühlingsruf am 16. I., 1916 am 2. II., 1917 am 11. II. Ich sah sie auch Mauern und Grabsteine nach Insekten absuchen. Die stimmliche Variationsfähigkeit dieser Art ist auffallend. Am 9. VI. 17. hörte ich im Priebischer Bruch eine Kohlmeise, deren Ruf so abweichend war, dafs ich mich erst durch den Augenschein von der Art überzeugen mufste. Der Ruf klang wie $w^{w \ddot{w}}{ }^{w i}{ }^{w i}{ }_{w u ̈}{ }^{w i}$, der Ton auf der zweiten, tiefer liegenden Silbe - und hatte einen melancholischen, klagenden Charakter, ganz im Gegensatz zu den sonstigen kecken und lebhaften Rufen dieser Art.

37. Sitta caesia Wolf. Der K l e i b e r ist hier ein recht spärlicher Brutvogel im Eichenbestand des Kankeler Waldes Welcher Form die Exemplare angehören, konnte ich aus Mangel an Belegexemplaren nicht feststellen, nehme aber an, dafs sie zur Form sordida Rchw. gehören.

38. Certhia familiaris L. und Certhia familiaris brachydactyla Brehm. Der B a u mläufer ist hier ziemlich häufig. In der Promenade, in gröfseren Gärten, besonders in einer jetzt in der Abholzung begriffenen Allee von alten Bäumen, besonders Akazien, die nach dem Schiefswerderwalde führt. Im Kiefernwald traf ich ihn nur an Stellen mit eingesprengtem Laubolz. Ein am 28. IV. 16. von Woynowitz eingeliefertes Stück mafs Rostrum a. fr. 16, Hinterkralle $6,5 \mathrm{~mm}$. Hiernach, sowie nach der Rückenfärbung gehörte es unzweifelhaft zu brachydactyla. Andrerseits traf ich manchmal Exemplare mit mehr Gelb auf dem Rücken und bisweilen - z. B. am 7. I. 1916 - sogar auffallend hell- und gelbrückige Stücke, über deren Zugehörigkeit zur typischen familiaris kein Zweifel sein konnte. Im Übrigen verweise ich, um Wiederholungen zu vermeiden, auf meine Arbeit uber den Baumläufer in Orn. Monatsber. 1918 S. 81 ff. 
39. Alauda arvensis L. Die F e l d l e r $\mathrm{ch}$ e ist hier häufig. 1916 Ankunft am 22. II. Am 3. X. 17. erhielt ich ein an einem Draht verletztes, flugunfähiges Stück. - Am 12. IV. 18. fand ich im Schiefswerderwalde Reste einer zerrissenen Feldlerche.

40. Lullula arborea L. Die He idele $\mathrm{rche}$ ist hier ziemlich häufig auf den Schlägen des Reisener Waldes, auf denen die jungen Kiefern noch klein sind und lückenhaft stehen, sowie an sonstigen lückenhaften Stellen der benachbarten Waldungen mit sandigem Boden. 1914 erster Gesang am 18. III., 1916 am 26. III., 1918 am 11. III.

41. Galerida cristata L. Die H a u ben lerche ist hier recht häufig; was wohl - abgesehen von der ihr zusagenden Bodenbeschaffenheit - mit den zahlreichen Exerzierplätzen, Sandgruben und ähnlichen Örtlichkeiten zusammenhängt, andrerseits auch mit dem regen Wagenverkehr, namentlich an Markttagen (Rofsäpfel). Am 18. I. 14. zählte ich an einem und demselben Strafsenteil 9 Stück. Sie suchen hier auch während der wärmeren Jahreszeit auf den Strafsen ihr Futter.

42. Budytes flavus L. Die $\mathrm{Ku} \mathrm{h} \mathrm{s} \mathrm{te} \mathrm{lz}$ e ist hier an geeigneten Örtlichkeiten in mäfsiger Zahl vertreten, so auf den Wiesen bei Kloda, an der Kostener Chausse u. s. w.

43. Motacilla boarula L. Die Gebirgs ba ch stelzo habe ich hier ein einziges Mal in 2 Exemplaren am 21. IX. 1914 an dem Teich auf dem hiesigen Schlofsplatz beobachtet.

44. Motacilla alba L. Die we ifse Bachstelze ist hier in der Nähe der Stadt eine Seltenheit, nur zuweilen erscheint sie an den hiesigen künstlich angelegten Teichen. Dagegen trifft man sie häufiger in der Nähe der in wasserreicher Umgebung gelegenen Ortschaften wie Tharlang, Lindensee u. s. w. Ankunft 1915 am 28. III., 1916 am 31. III., 1917 am 28. III., 1918 am 24. III., nachdem Herr Lenhard schon am 22. III. eine an einem Draht verunglückte erhalten hatte.

45. Anthus pratensis L. Der W i s e n pie per kommt auf den Wiesen im Priebischer Bruch, sowie im Kraschener Bruch und an anderen Örtlichkeiten in spärlicher Zahl vor. 1916 sah ich ihn im Kraschener Bruch schon am 6. IV. Am 3. IV. 1917 wurde ein am Draht erstofsener zum Ausstopfen eingeliefert.

46. Anthus trivialis L. Der Ba $\mathrm{Bmpieper}$ hat im Tharlanger Bruch infolge der Abholzungen (s. oben unter Tharlanger Bruch) und der dort betriebenen Wiesenkultur aufserordentlich zugenommen. Hiervon abgesehen kommt er in hiesiger Gegend nicht häufig vor.

(Schlufs folgt.) 


\section{$2 \mathrm{BHL}$ Biodiversity Heritage Library}

Kayser, Charles. 1920. "Die vögel der umgebung von lissa i./P." Journal $f u$

r Ornithologie 68, 326-340. https://doi.org/10.1007/bf02537890.

View This Item Online: $\underline{\text { https://www.biodiversitylibrary.org/item/43506 }}$

DOI: https://doi.org/10.1007/bf02537890

Permalink: https://www.biodiversitylibrary.org/partpdf/141107

\section{Holding Institution}

MBLWHOI Library

\section{Sponsored by}

MBLWHOI Library

\section{Copyright \& Reuse}

Copyright Status: No known copyright restrictions as determined by scanning institution.

This document was created from content at the Biodiversity Heritage Library, the world's largest open access digital library for biodiversity literature and archives. Visit BHL at https://www.biodiversitylibrary.org. 